\title{
Características das Tecnologias Digitais da Indústria 4.0 que têm apoiado a
}

\section{Economia Circular}

\author{
Characteristics of Industry 4.0 Digital Technologies that have supported the Circular Economy
}

Características de la Tecnología Digital de la Industria 4.0 que han apoyado la Economía Circular

Recebido: 06/11/2021 | Revisado: 16/11/2021 | Aceito: 29/11/2021 | Publicado: 11/12/2021

Elcio Ricardo Leite Guimarães

ORCID: https://orcid.org/0000-0002-1410-7744 Autarquia Municipal de Previdência e Assistência à Saúde do Servidor do Recife, Brasil E-mail: elcio.guimaraes@upe.br

Djalma Silva Guimarães Júnior ORCID: https://orcid.org/0000-0002-4539-8492 Universidade de Pernambuco, Brasil E-mail: Djalma.guimaraes@upe.br

\begin{abstract}
Resumo
A história tem dado alguns saltos de tempos em tempos, quando o assunto é evolução, com mudanças de aspectos organizacionais relacionados à sistemas de produção, processos administrativos, planejamento, definição de missão e valores que norteiam as boas práticas administrativas, para atendimento aos anseios da sociedade. São muitos os conceitos e teorias que caracterizam tais mudanças e eles variam de acordo com a realidade que se vive na época de suas definições. Nos tempos atuais, muito se tem falado sobre Indústria 4.0 e sobre Economia Circular - EC, e suas respectivas importâncias para o alcance de um desenvolvimento calcado no triplo botton line - TBL, que busca o melhor equilíbrio entre os fatores econômicos, sociais e ambientais. Este estudo tem por objetivo realçar parte dos trabalhos que tenham explorado tal conhecimento, sob a ótica das possíveis contribuições que a Indústria 4.0 esteja oferecendo para uma efetiva implementação de EC no âmbito das organizações. Através de uma revisão integrativa fez-se a pesquisa para identificar esses estudos, apoiando-se na técnica de análise de conteúdo de Bardin (1977), tendo-se obtido êxito na elaboração de uma coletânea de trabalhos encontrados no ambiente científico, que certamente nortearão quaisquer pesquisas referentes a essa temática. Os resultados apontam para uma atenção cada vez maior às questões tecnológicas e sua importante integração com o desenvolvimento sustentável, chancelando a percepção da constante atualização dos modelos de negócios e das teorias de gestão, em sintonia com a realidade atual.
\end{abstract}

Palavras-chave: Indústria 4.0 e economia circular; Economia circular nas organizações.

\begin{abstract}
History has taken some leaps from time to time, when the subject is evolution, with changes in organizational aspects related to production systems, administrative processes, planning, mission definition and values that guide good administrative practices, to meet society's wishes. There are many concepts and theories that characterize such changes, and they vary according to the reality that is lived at the time of their definitions. Nowadays, much has been said about Industry 4.0 and Circular Economy - EC, and their respective importance for achieving a development based on the triple bottom line - TBL, which seeks the best balance between economic, social, and environmental factors. This study aims to highlight part of the works that have explored such knowledge, from the perspective of the possible contributions that Industry 4.0 is offering for an effective implementation of CE within organizations. Through an integrative review, the research was carried out to identify these studies, based on Bardin's (1977) content analysis technique, with success in the preparation of a collection of works found in the scientific environment, which will certainly guide any related research. to this theme. The results point to an increasing attention to technological issues and their important integration with sustainable development, confirming the perception of the constant updating of business models and management theories, in tune with the current reality.
\end{abstract}

Keywords: Industry 4.0 and circular economy; Circular economy in organizations.

\section{Resumen}

La historia ha dado algunos saltos de vez en cuando, cuando el tema es la evolución, con cambios en los aspectos organizativos relacionados con los sistemas de producción, los procesos administrativos, la planificación, la definición de misión y los valores que orientan las buenas prácticas administrativas, para satisfacer las expectativas de la sociedad. Son muchos los conceptos y teorías que caracterizan tales cambios y varían según la realidad que se vive al momento de sus definiciones. Hoy en día se ha hablado mucho sobre la Industria 4.0 y la Economía Circular - CE, y 
su respectiva importancia para lograr un desarrollo basado en el triple resultado - TBL, que busca el mejor equilibrio entre factores económicos, sociales y ambientales. Este estudio tiene como objetivo resaltar parte de los trabajos que han explorado dicho conocimiento, desde la perspectiva de los posibles aportes que la Industria 4.0 está ofreciendo para una implementación efectiva de la CE en las organizaciones. A través de una revisión integradora, se llevó a cabo la investigación para identificar estos estudios, basados en la técnica de análisis de contenido de Bardin (1977), con éxito en la elaboración de una colección de trabajos encontrados en el ámbito científico, que sin duda guiarán cualquier investigación relacionada con esta temática. Los resultados apuntan a una creciente atención a las cuestiones tecnológicas y su importante integración con el desarrollo sostenible, confirmando la percepción de la constante actualización de los modelos de negocio y teorías de gestión, en sintonía con la realidad actual.

Palabras clave: Industria 4.0 y economía circular; Economía circular en las organizaciones.

\section{Introdução}

A humanidade vem perpassando diversas transformações sociais, com profundas mudanças nos campos tecnológicos, econômicos e ambientais (Ghobakhloo, 2020). Dentre tais transformações surge o conceito de Indústria 4.0 que, embora inicialmente seja difuso, vem agregando tecnologias capazes de enfrentar melhor o desafio de equilibrar o desenvolvimento econômico, social e ambiental, em uma "visão sociotécnica" (Beier et al., 2020). As transformações que passaram pela fase de mecanização, uso de energias tradicionais e alternativas, das telecomunicações, sistemas de tecnologia e informação para a atual fase que compreende a internet das coisas e objetos - IoT, banco de dados - Big Data, robotização, uso de armazenamento e comunicação em nuvens, dentre outras, são verdadeiras revoluções para o setor público e privado (Ardito et al., 2019; Buer et al., 2018; Schroeder et al., 2019). Surgem novas abordagens que tanto trazem as questões tecnológicas, como comportamentais e requisitam atitudes equilibradas entre o desenvolvimento econômico, social e ambiental, com ênfase para a sustentabilidade e para a Economia Circular - EC.

Este trabalho aborda o tema da Economia Circular - EC, no nível organizacional, haja vista não se tratar de matéria antiga, porém, uma temática das últimas décadas, conforme dito por Niero e Hauschild (2017). Mendoza et al., (2017) desenvolveram uma estrutura para que as empresas possam enfrentar a EC de forma holística, incorporando o princípio de que o processo decisório que leva aos resultados esperados pela união entre indústria 4.0 e EC, precisa ser implementado no nível corporativo, para que sua adoção obtenha o êxito esperado. Nesse sentido, encontra-se também a contribuição do modelo proposto pela British Standard (2017), denominado de BS 8001, com o título "Framework for implementing the principles of the circular economy in organizations", que fornece um padrão de diretrizes para a "transição de organizações em direção a um modelo de operação mais circular e sustentável.” A British Standard propõe uma composição estrutural flexível de oito estágios para a melhoria contínua.

Para que haja sustentabilidade na aplicabilidade do conceito de economia circular, sob os aspectos econômicos, sociais e ambientais, Niero e Hauschild (2017) propõem a utilização do "modelo de Avaliação de Sustentabilidade do Ciclo de Vida - LCSA, por sua maior abrangência e aplicação operacional.”

No padrão proposto pela British Standard BS 8001-2017, modelo padrão que se assemelha à norma ISO 14040, a EC é definida como "aquela economia regida por princípios restaurativos e regenerativos, que visa manter produtos, componentes e materiais em seus máximos valores de utilidade, distinguindo os ciclos técnicos e biológicos.” Este conceito se caracteriza de duas formas: i) envolver todos os níveis e mover a organização em direção à sustentabilidade; ii) criar valor via inovação em produtos, serviços e modelo de negócio.

Essas formas ocorrem através de processos decisórios e pela adoção de instrumentos de integração de estruturas para que se aplique o modelo de EC. (Pigosso \& Rozenfeld, 2013).

De acordo com TET Dantas et.al. (2020), há dois tópicos relevantes que podem dar contribuições significativas para a implementação dos 17 Objetivos Sustentáveis nas Organizações, que são: a Economia Circular (EC) e a Indústria 4.0. A 
avaliação de nexos entre esses tópicos emergentes, permitiu identificar a influência direta nos Objetivos de Desenvolvimento Sustentáveis - ODS 7, 8, 9, 11, 12 e 13, pois faz a conexão de tecnologia inovadora e aspectos de EC para sua aplicabilidade em favor da sustentabilidade.

Na busca por teorias e definições que efetivamente possam relacionar a melhoria sistêmica às funções modernas da tecnologia com consequências na EC, afirma Eivind et al (2020), que as tecnologias digitais são responsáveis pelo aumento da produtividade e da eficiência. Os tópicos abordados passam por uma revisitação aos conceitos de EC, de Indústria 4.0 e da explanação sobre os estudos que abordam as possíveis contribuições deste para aquele conceito.

O mundo acadêmico, empresarial e organizacional vislumbra o melhor desenvolvimento de teorias para a implementação do conceito de Economia Circular, tornando-se uma exigência cada vez maior da sociedade moderna, por soluções que de fato aperfeiçoem a qualidade de vida e propiciem o desenvolvimento econômico, social e ambiental. Esta exigência tem pressa e é preciso se tornar prática a aplicabilidade do conceito de Economia Circular e como pode ser tal conceito implantado de forma mais ágil para trazer as soluções desejadas. Desta forma, o objetivo da pesquisa é verificar os estudos que permitem a integração da temática da EC com a da Indústria 4.0 com o propósito do desenvolvimento sustentável, revisando suas teorias e verificando que objetivos estratégicos podem ser viabilizados, através da junção desses dois importantes conceitos. Então, pergunta-se: quais tecnologias da Indústria 4.0 permitem a melhor aplicação da EC pelas organizações?

Reunir os conceitos existentes no campo da Indústria 4.0, das tecnologias digitais e da Economia Circular, numa formatação que permita a sua aplicabilidade nas organizações, servirá para facilitar o estudo e aprofundamento desse tema, contribuindo para os diversos públicos que nele possa se interessar. O trabalho pretende verificar a crescente investigação das práticas da indústria 4.0 sobre a Economia Circular.

Para contextualizar, os conceitos adotados estão caraterizados da forma como os autores pesquisados, mais predominantemente os descreveram. Nesse sentido, em relação à Indústria 4.0, entende-se que as novas tecnologias vêm propiciando uma nova revolução, onde pode-se destacar as inovações na área das energias renováveis, as perspectivas abertas pela internet e pela digitalização que podem explicar parte das mudanças em curso, conforme preceitua Weetman (2019). Nesse contexto, surgem novos conceitos, a exemplo de “inovação frugal”, que é uma forma disruptiva de criar negócios com maiores valores sociais sendo mais eficazes e efetivos. (Weetman, 2019).

Apesar de existirem várias definições sobre o significado de Indústria 4.0, a maioria delas leva a conceitos que perpassam visões sobre modernização nas áreas de logística, abastecimento, processos produtivos, autossuficiência na produção, prolongamento do tempo de vida de produtos e serviços, bem como o uso integrado de tecnologias que permitam a menor dependência em relação ao ser humano. (Castelo-Branco et al., 2019).

De acordo com os oito estudos destacados adiante, com pontuação máxima na tabela 4 deste artigo, pode-se perceber que as tecnologias mais citadas pelos autores das obras ali descritas são: Internet das Coisas - IoT, Internet de Serviços - IoS, computação em nuvem, Big Data, Sistemas Cibernéticos Físicos - CPS, Inteligência Artificial - AI, Sistemas Ciber-Físicos de Produção - CPPS, design virtual, blockchain, impressão 3D, robótica, nanotecnologia, tecnologias de visualização (realidade virtual e aumentada), manufatura aditiva, digital gêmeos.

Em relação ao conceito predominante de Economia Circular, sob a ótica dos autores pesquisados, percebe-se que há uma reivindicação social crescente em relação a melhor equilíbrio entre os fatores ligados ao desenvolvimento econômico, o desenvolvimento social e a sustentabilidade, traduzido pela expressão cravada pela comissão sobre o meio ambiente da Organização Mundial das Nações Unidas, que em 1987 na produção do Relatório de Brundtland definiu desenvolvimento 
sustentável como aquele que "satisfaz as necessidades do presente sem comprometer a capacidade de as futuras gerações satisfazerem as suas próprias necessidades.” (Rodrigues et al., 2015).

De acordo com o World Economic Forum (2014), a população mundial vem crescendo de forma exponencial e atingirá em 2030 o número de 9 bilhões de habitantes, dos quais 3 bilhões aptos para o consumo. As consequências que se impõe são: volatilidade de custos, inclusive dos recursos naturais; demanda maior que a oferta; escassez de insumos e de recursos; novas e maiores exigências por tecnologias socialmente inclusivas. Ficou estimado que em 2014 a população já consumia mais de uma vez e meia, por ano, os recursos disponíveis no planeta, em função de nos últimos 150 anos ter se adotado o sistema de economia linear, regida por: "produzir, consumir e descartar. Fator que ocasionou a sobrecarga ecológica no planeta."

De acordo com Cayzer et al. (2017), diversos estudos sobre a EC não deixam muito claro os níveis possíveis de sua importante contribuição. Os níveis podem ter a seguinte classificação: micro, meso e macro. Onde o micro representa as empresas que buscam as melhorias de seus próprios processos e desenvolvimento de negócios, de acordo com Ormazabal et al. (2018). O meso representa a percepção de EC em benefício da economia regional e a preservação da natureza, sob a ótica de Geng et al. (2012). E, o nível macro se traduz na promoção da sustentabilidade da sociedade, nas cidades, regiões e países, através de políticas ambientais e da influência institucional de governos e organizações, havendo uma concordância entre os quatro autores citados, em relação aos conceitos aqui disponibilizados. (Cayzer et al., 2017).

Nesse sentido, surgem as abordagens da economia circular, tais como: diminuir o consumismo, usar recursos sustentáveis, trocar o descarte por eliminação de resíduos e potencializar o design de produtos, a logística reversa, mais serviços e novas formas de comportamento organizacional, baseada nas ações de renovar, reparar e manufaturar. (Weetman \& Catherine, 2019).

Então, vislumbra-se como objetivo, a necessidade em destacar estudos que estejam contribuindo para uma melhor percepção das contribuições possíveis da indústria 4.0 e da digitalização em prol da aplicabilidade da economia circular, de forma efetiva, rumo ao desenvolvimento sustentável.

\section{Metodologia}

Entre os métodos de análise qualitativa, o presente estudo adota a técnica de análise de conteúdo como forma de abordar o levantamento, descrição, organização, critérios de escolha e apresentação de resultados sobre o tema pesquisado. Como alegado por Freitas et al. (1997), a análise de conteúdo requer um esforço concentrado e alta disciplina por parte do pesquisador para exercer as etapas de definição de categorias, efetuar análises e ter rigor técnico na formulação do estudo.

Para tanto, adota-se os princípios estabelecidos por Bardin (2006), cuja técnica consiste numa análise de dados utilizada em pesquisas qualitativas, por isso afirma Flick (2009), que é necessário que seja bem definida a metodologia e o programa a ser seguido e, acrescenta Creswell (2007), que este método requer muita atenção e capacidade de interpretação do pesquisador.

Trata-se, portanto, de uma Revisão Integrativa sobre artigos, estudos, obras acadêmicas e trabalhos relacionados aos temas que demonstrem as possíveis contribuições da Indústria 4.0 para a EC com repercussões no alcance dos Objetivos de Desenvolvimento Sustentável - ODS. A busca procura vislumbrar os elementos que permeiem o fluxo delineado na Figura 1: 
Figura 1. Fluxo da classificação, categorização e codificação da análise.

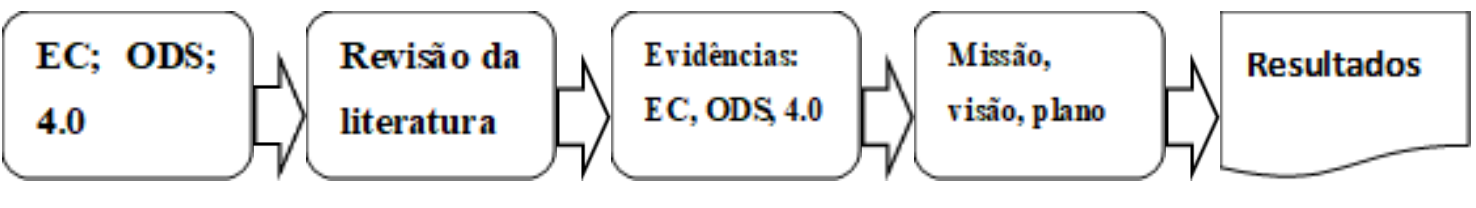

Fonte: Autores.

O fluxo da Figura 1 explica a sequência da revisão integrativa da literatura, cuja definição de critérios abaixo explicados, permitirá encontrar as evidências em estudos que relacionem a temática abordada, verificando se tais práticas estão incorporadas nas organizações desde o seu nível estratégico até o operacional e, por fim, se os resultados são constatadores de sua efetiva prática organizacional.

De acordo com Bardin (2006), as fases para a verificação de dados em análise de conteúdo são: i) pré-análise; ii) exploração do material; iii) tratamento dos resultados, inferência e interpretação. Na fase da pré-análise é feita a catalogação dos dados, através da "leitura flutuante" e em seguida é feita a formulação das hipóteses e objetivos para que se defina os indicadores. A fase de exploração do material consiste na definição das categorias para cada conteúdo alvo de análise e a verificação das suas respectivas frequências de ocorrência nos achados. Dessa forma, os elementos mais expoentes dessa fase são: “codificação, classificação e categorização." $\mathrm{Na}$ fase de resultados, inferência e interpretação se faz a condensação e destaque das informações de conteúdo, seguida das inferências interpretativas e das análises reflexiva e crítica de todo o material selecionado.

Através de critérios previamente estabelecidos, é possível dar sequência à etapa de resultados. Desta forma, a presente pesquisa será pautada pela análise de conteúdo proposta na técnica desenvolvida por Bardin (2006), definindo os parâmetros necessários ao desenvolvimento das três fases citadas anteriormente, conforme proposto na Tabela 1.

Tabela 1. Categorias de análise do corpus de texto.

\begin{tabular}{|c|c|c|c|c|}
\hline 1. Aborda & $\begin{array}{r}\text { em da EC no âmbito } \\
\text { das organizações }\end{array}$ & $\begin{array}{r}\text { 2. Aplica } \\
\text { estrutu }\end{array}$ & $\begin{array}{l}\text { e modelos } \\
\text { de EC-TBL }\end{array}$ & \multirow[t]{2}{*}{$\begin{array}{l}\text { 3. Resultados } \\
\text { obtidos em EC. }\end{array}$} \\
\hline \multicolumn{2}{|l|}{ 1.1 Subcategorias } & \multicolumn{2}{|c|}{ 2.1 Subcategorias } & \\
\hline $\begin{array}{l}\text { 1.1.1 EC holística } \\
\text { caracterizada na } \\
\text { forma transversal. }\end{array}$ & $\begin{array}{l}\text { 1.2.1 EC no nível } \\
\text { corporativo de } \\
\text { decisão. }\end{array}$ & $\begin{array}{l}\text { 2.1.1Usa } \\
\text { tecnologias } \\
\text { inovadoras em } \\
\text { seus modelos. }\end{array}$ & $\begin{array}{l}\text { 2.2.1 As } \\
\text { tecnologias estão } \\
\text { focadas nos } 17 \\
\text { ODS. }\end{array}$ & $\begin{array}{l}\text { 3.1 Quantas e quais } \\
\text { tecnologias } \\
\text { inovadoras em uso. }\end{array}$ \\
\hline $\begin{array}{lr}1.1 .2 \text { Áreas } & \text { da } \\
\text { organização que se } \\
\text { envolvem } & \text { na } \\
\text { implementação } & \text { da } \\
\text { EC. } & \end{array}$ & $\begin{array}{l}1.2 .2 \text { EC } \\
\text { incorporada na } \\
\text { missão e praticada } \\
\text { na organização. }\end{array}$ & $\begin{array}{l}2.1 .2 \mathrm{As} \\
\text { tecnologias que } \\
\text { favorecem a } \\
\text { criação e } \\
\text { preservação de } \\
\text { indicadores EC. }\end{array}$ & $\begin{array}{l}2.2 .2 \mathrm{O} \text { modelo foi } \\
\text { sistematizado e } \\
\text { possui metas de } \\
\text { resultados para EC. }\end{array}$ & $\begin{array}{l}\text { 3.2 Quantos e quais } \\
\text { objetivos ODS com } \\
\text { metas definidas no } \\
\text { planejamento. }\end{array}$ \\
\hline
\end{tabular}

Fonte: Autores, com base na leitura flutuante.

$\mathrm{Na}$ análise do material coletado, se estabelece o valor 1 (um) para as categorias achadas no processo de leitura e 0 (zero) para a ausência da categoria no material, selecionando-se assim o que é alvo do aprofundamento do estudo para embasamento, discussão e conclusão. Para a subcategoria se estabelece o valor 0,5 (meio) quando ela está presente e 0 (zero) para a sua ausência nos documentos em pesquisa. Dessa forma se conduz a metodologia, incluindo-se os procedimentos de 
avaliação e controle, necessários para corrigir eventuais desvios de análise e decisão. Dos achados com pontuação se realiza a leitura para se verificar a aderência ou não às categorias e subcategorias definidas na Tabela 1. Os artigos sem aderência são descartados e os que possuem aderência são avaliados com base nos fatores de exaustividade, representatividade, homogeneidade e pertinência. A exaustividade revela a profundidade revelada em relação ao foco deste estudo, qual seja: a efetiva contribuição do artigo sobre as tecnologias da Indústria 4.0 que são capazes de apoiar a melhor e mais efetiva aplicabilidade da EC nas organizações. Dessa mesma forma se analisa os demais fatores de aderência no conteúdo, conforme citados acima. Trata-se, portanto de uma análise de caráter interpretativa, incorporando assim uma parte subjetiva do critério de análise ora apresentado, porém, baseada numa seleção objetiva dos achados, em função dos parâmetros anteriormente definidos. (Bardin, 1977).

\section{Resultados e Discussão}

Com o termo de busca no Science Direct sendo ("Circular Economy") AND (“Industry 4.0") foram achados para o período entre 2019 e 2021 o total de 660 artigos, após refinar para conter apenas artigos revisados por pares em ciência do meio ambiente, restaram 41, cuja leitura foi aprofundada para seleção dos artigos contemplados nos critérios, categorias e subcategorias anteriormente definidas. A Tabela 2 demonstra o resultado da busca refinada.

Tabela 2. Seleção de artigos para análise de conteúdo.

\begin{tabular}{|c|c|c|c|c|c|c|c|}
\hline \multirow{2}{*}{$\begin{array}{l}\begin{array}{l}\text { Tipo de } \\
\text { artigo: }\end{array} \\
\text { Revisados }\end{array}$} & \multirow{2}{*}{$\begin{array}{l}\text { Área: } \\
\text { Ciência } \\
\text { ambiental }\end{array}$} & \multicolumn{3}{|c|}{ Tema/título da publicação: } & \multirow[t]{2}{*}{2019} & \multirow[t]{2}{*}{2020} & \multirow[t]{2}{*}{2021} \\
\hline & & $\begin{array}{l}\text { Produção } \\
\text { limpa }\end{array}$ & $\begin{array}{l}\text { Recursos, } \\
\text { conservação e } \\
\text { reciclagem }\end{array}$ & $\begin{array}{l}\text { Gerenciamento de } \\
\text { resíduos }\end{array}$ & & & \\
\hline 41 & 30 & 26 & 2 & 2 & 8 & 9 & 21 \\
\hline
\end{tabular}

Fonte: Autores (2021), a partir de dados do Science Direct.

A pesquisa foi primeiramente refinada para a descoberta de artigos que fossem revisados por pares e publicados na área de ciência ambiental. A plataforma então gerou 41 estudos revisados, dos quais 30 na área de ciência ambiental e identificou que desses 30, exatamente 26 se referiam a Produção Limpa; 2 a Recursos, Conservação e Reciclagem e; 2 a Gerenciamento de Resíduos. Interessante a constatação da evolução quantitativa na série temporal usada, que apontou 8 estudos em 2019, 9 em 2020 e 21 em 2021. Cabe o esclarecimento de se ter definido esse horizonte temporal, em função de se querer analisar aspectos da modernidade tecnológica da indústria 4.0 que favorecem a EC, portanto não seria pertinente obter dados mais longínquos que correriam o risco de não contemplarem as tecnologias habilitadoras da Indústria 4.0.

Dos 30 artigos com a temática referente às palavras de busca utilizada, 5 não se relacionavam com o objetivo deste trabalho e dos 25 que se relacionavam, através da leitura se identificou que 14 tinham aderência às duas categorias simultaneamente e desses, 11 também aderiam à subcategoria um e 11 à subcategoria dois. Dos que não tinham adesão simultânea às duas categorias, constatou-se que 5 aderiam apenas à categoria um, que 3 aderiam apenas à categoria dois e 3 não tiveram adesão à nenhuma categoria. Cujas quantidades em relação às categorias e às subcategorias estão apresentadas na Tabela 3 . 
Research, Society and Development, v. 10, n. 16, e186101622912, 2021

(CC BY 4.0) | ISSN 2525-3409 | DOI: http://dx.doi.org/10.33448/rsd-v10i16.22912

Tabela 3. Quantidade de artigos por categoria e subcategoria definidas.

\begin{tabular}{llll}
\hline & TOTAL & Subcategoria 1 & Subcategoria 2 \\
\hline Duas categorias & 14 & 11 & 11 \\
\hline Só categoria um & 05 & 01 & 01 \\
\hline Só categoria dois & 03 & & \\
\hline Nenhuma & 03 & & \\
\hline subtotal & 25 & & \\
\hline Fora do escopo & 05 & & \\
\hline TOTAL & 30 & & \\
\hline
\end{tabular}

Fonte: Autores.

Através da pontuação atribuída às categorias e subcategorias, temos a seguinte pontuação para os achados da pesquisa, conforme Tabela 4:

Tabela 4. Pontuação dos estudos achados por categorias e subcategorias.

\begin{tabular}{|c|c|c|c|c|}
\hline Estudo & Autor/ano & $\begin{array}{l}\text { Aderência às } \\
\text { categorias }\end{array}$ & $\begin{array}{l}\text { Aderência às } \\
\text { subcategorias }\end{array}$ & Pontuação \\
\hline $\begin{array}{l}\text { Industry } 4.0 \text { and sustainable development: A } \\
\text { systematic mapping of triple bottom line, Circular } \\
\text { Economy and Sustainable Business Models } \\
\text { perspectives. }\end{array}$ & $\begin{array}{l}\text { Iqra Sadaf Khan; Muhamada } \\
\text { Ovais Ahmad; Jukka Majava. } \\
\qquad 2021 .\end{array}$ & 1 e 2 & 1.1 e 2.1 & 3 \\
\hline $\begin{array}{l}\text { How the combination of Circular Economy and } \\
\text { Industry } 4.0 \text { can contribute towards achieving the } \\
\text { Sustainable Development Goals. }\end{array}$ & $\begin{array}{l}\text { T E T Dantas; E. D. de Souza; } \\
\text { S. R. Soares. } 2020 .\end{array}$ & 1 e 2 & 1.1 e 2.1 & 3 \\
\hline $\begin{array}{l}\text { Potentials of industry } 4.0 \text { for supply chain } \\
\text { management within the triple bottom line of } \\
\text { sustainability }- \text { A systematic literature review. }\end{array}$ & $\begin{array}{l}\text { Hendrik Birkel e Julian M. } \\
\text { Müller. } 2020 .\end{array}$ & 1 e 2 . & 1.1 e 2.1 & 3 \\
\hline $\begin{array}{l}\text { Industry } 4.0 \text { and sustainability: Towards } \\
\text { conceptualization and theory. }\end{array}$ & $\begin{array}{l}\text { Mirjam Beltrami; Guido Orzes } \\
\text { e Marco Sartor. } 2021 .\end{array}$ & 1 e 2 . & 1.1 e 2.1 & 3 \\
\hline $\begin{array}{l}\text { The circular chemistry conceptual framework: A way } \\
\text { forward to sustainability in industry } 4.0 \text {. }\end{array}$ & $\begin{array}{c}\text { S. Venkata Mohan e } \\
\text { Ranaprathap Katakojwala. } \\
2020 . \\
\end{array}$ & 1 & & 1 \\
\hline $\begin{array}{l}\text { Industry } 4.0 \text { ten years on: A bibliometric and } \\
\text { systematic review of concepts, sustainability value } \\
\text { drivers, and success determinants. }\end{array}$ & $\begin{array}{l}\text { Morteza Ghobakhloo; Massod } \\
\text { Fathi e Manuel E. Morales. } \\
2021 .\end{array}$ & 1 e 2 & 1.1 e 2.1 & 3 \\
\hline $\begin{array}{l}\text { A review of emerging industry } 4.0 \text { technologies in } \\
\text { remanufacturing. }\end{array}$ & $\begin{array}{l}\text { Mairi Kerin e Duc Truong } \\
\text { Pham. } 2019 .\end{array}$ & 1 e 2. & 1.1 e 2.1 & 3 \\
\hline $\begin{array}{l}\text { Digitalisation and intelligent robotics in value chain of } \\
\text { circular economy oriented waste management - A } \\
\text { review }\end{array}$ & $\begin{array}{l}\text { R. Sarc; A. Curtis e R. } \\
\text { Pmberger. } 2019 .\end{array}$ & 1 e 2. & 1.1 e 2.1 & 3 \\
\hline $\begin{array}{l}\text { Industry 4.0, digitization, and opportunities for } \\
\text { sustainability. }\end{array}$ & Morteza Ghobakhloo. 2019. & 1 e 2. & 1.1 & 2,5 \\
\hline $\begin{array}{l}\text { 'Unlocking circular economy for prevention of marine } \\
\text { plastic pollution: An exploration of G } 20 \text { policy and } \\
\text { initiatives'. }\end{array}$ & $\begin{array}{l}\text { Zinaida Fadeva e Rene Van } \\
\text { Berkel. } 2020 .\end{array}$ & 1 & 1.1 & 1,5 \\
\hline $\begin{array}{l}\text { Plastic recycling in additive manufacturing: A } \\
\text { systematic literature review and opportunities for the } \\
\text { circular economy. }\end{array}$ & $\begin{array}{l}\text { Fabio A. Cruz Sanchez Hakim } \\
\text { Boudaoud e Joshua M. Pearce. } \\
\qquad 2020 .\end{array}$ & 1 e 2 & 1.1 e 2.1 & 2 \\
\hline $\begin{array}{l}\text { Transitioning China to a circular economy through } \\
\text { remanufacturing: A comprehensive review of the } \\
\text { management institutions and policy system. }\end{array}$ & $\begin{array}{l}\text { Xueliang Yuan; Mengyue Liu } \\
\text { e Jian Zuo. } 2020 .\end{array}$ & 1 & & 1 \\
\hline $\begin{array}{l}\text { A literature review on circular economy adoption in } \\
\text { the manufacturing sector. }\end{array}$ & $\begin{array}{l}\text { Federica Acerbi e Marco } \\
\text { Taisch. } 2020 .\end{array}$ & 1 e 2 & 1.1 e 2.1 & 3 \\
\hline $\begin{array}{l}\text { Reuse assessment of WEEE: Systematic review of } \\
\text { emerging themes and research directions }\end{array}$ & $\begin{array}{l}\text { Gurunathan Anandh, } \\
\text { Shanmugam Prasanna } \\
\text { Venkatesan e Kaliyan } \\
\text { Mathiyazhagan. } 2021 .\end{array}$ & 2 & 2.1 & 1,5 \\
\hline
\end{tabular}




\begin{tabular}{|c|c|c|c|c|}
\hline $\begin{array}{l}\text { A triple bottom line examination of product } \\
\text { cannibalisation and remanufacturing: A review and } \\
\text { research agenda. }\end{array}$ & $\begin{array}{l}\text { Okechukwu Okorie, Martins } \\
\text { Obi e Konstantinos Salonitis. } \\
2021 .\end{array}$ & 2 & & 1 \\
\hline $\begin{array}{l}\text { Standardisation: An essential enabler for the circular } \\
\text { reuse of construction components? A trajectory for a } \\
\text { cleaner European construction industry. }\end{array}$ & $\begin{array}{l}\text { K. Anastasiades, J. Goffin e J. } \\
\text { Blom. } 2021 .\end{array}$ & 1 & & 1 \\
\hline $\begin{array}{l}\text { Driving vehicle dismantling forward - A combined } \\
\text { literature and empirical study. }\end{array}$ & $\begin{array}{c}\text { Malin Tarrar, Mélanie } \\
\text { Despeisse e Björn Johansson. } \\
2021 .\end{array}$ & 1 & & 1 \\
\hline $\begin{array}{l}\text { Circular supply chain management: A definition and } \\
\text { structured literature review. }\end{array}$ & $\begin{array}{c}\text { Muhammad Farooque, } \\
\text { Abraham Zhang e Donald } \\
\text { Huisingh. } 2019 .\end{array}$ & 1 e 2 & 1.1 & 2,5 \\
\hline $\begin{array}{l}\text { Circular supply chain management: A state-of-art } \\
\text { review and future opportunities. }\end{array}$ & $\begin{array}{l}\text { Swapnil Lahane, Ravi Kant e } \\
\text { Ravi Shankar. } 2020 .\end{array}$ & $1 \mathrm{e} 2$ & 2.1 & 2,5 \\
\hline $\begin{array}{l}\text { Circular Business Models versus circular benefits: An } \\
\text { assessment in the waste from Electrical and Electronic } \\
\text { Equipments sector. }\end{array}$ & $\begin{array}{c}\text { Paolo Rosa, Claudio } \\
\text { Sassanelli e Sergio Terzi. } \\
2019 . \\
\end{array}$ & 1 e 2 & & 2 \\
\hline $\begin{array}{l}\text { Towards Circular Business Models: A systematic } \\
\text { literature review on classification frameworks and } \\
\text { archetypes. }\end{array}$ & $\begin{array}{c}\text { Paolo Rosa, Claudio } \\
\text { Sassanelli e Sergio Terzi. } \\
2019 . \\
\end{array}$ & 1 e 2 & 2.1 & 2,5 \\
\hline $\begin{array}{l}\text { Promoting sustainable cleaner production paradigms } \\
\text { in palm oil fuel ash as an eco-friendly cementitious } \\
\text { material: A critical analysis. }\end{array}$ & $\begin{array}{c}\text { Muhammad Ayub, Mohd } \\
\text { Hafiz Dzarfan Othman e } \\
\text { Juhana Jaafar. 2021. }\end{array}$ & Zero & Zero & Zero \\
\hline $\begin{array}{l}\text { The future of waste management in smart and } \\
\text { sustainable cities: A review and concept paper. }\end{array}$ & $\begin{array}{c}\text { Behzad Esmaeilian, Ben } \\
\text { Wang e Sara Behdad. } 2018 .\end{array}$ & 2 & & 1 \\
\hline $\begin{array}{l}\text { Circular business models: Current aspects that } \\
\text { influence implementation and unaddressed subjects. }\end{array}$ & $\begin{array}{c}\text { Rodrigo Salvador, Murillo } \\
\text { Vetroni Barros e Antonio } \\
\text { Carlos de Francisco. } 2019 .\end{array}$ & Zero & Zero & Zero \\
\hline $\begin{array}{l}\text { The Delft } 1974 \text { and } 2019 \text { European Loss Prevention } \\
\text { Symposia: Highlights and an impression of process } \\
\text { safety evolutionary changes from the } 1^{\text {st }} \text { to the } 16^{\text {th }} \\
\text { LPS. }\end{array}$ & $\begin{array}{l}\text { Hans J. Pasman e Bruno } \\
\text { Fabiano. } 2020 .\end{array}$ & Zero & Zero & Zero \\
\hline
\end{tabular}

Fonte: Autores.

É certo que a análise qualitativa fundamentada por Bardin, através da técnica de análise de conteúdo, com a utilização da leitura flutuante para a verificação da aderência dos estudos achados, tendo por base os critérios e categorias aqui definidos, permite caracterizar a assertividade da pesquisa sob a ótica do pesquisador, fato que confere um misto de objetividade e subjetividade ao estudo ora apresentado. Pode-se discutir a relevância ou o nível de confiança desse tipo de metodologia, quando comparado com os programas disponíveis para análise bibliométrica de pesquisas de natureza semelhante. A de se considerar, também, a importância da coerência para o pesquisador responsável pelas análises, pois os resultados dependem fundamentalmente de sua inferência e interpretação em relação aos parâmetros estabelecidos, o que exige muita dedicação, atenção e alto rigor discricionário na avaliação e pontuação dos achados. Nesse sentido, foi fundamental a escolha seletiva por estudos revisados por pares, em função da qualidade encontrada nos estudos com alto nível de aderência aos objetivos deste trabalho. Esses estudos denotam a crescente atenção pelo mundo acadêmico em relação ao tema sustentabilidade com base nos fatores tecnológicos que favorecem a EC. Essa constatação vem ao encontro dos anseios sociais por melhorias na qualidade de vida e pelo melhor uso dos recursos naturais, em um mundo em constante transformação, cuja dinâmica tem acelerado exponencialmente as mudanças organizacionais, implicando na necessidade de maior absorção de conhecimento, alteração de comportamentos e de atitudes, trazendo impactos positivos e negativos para a humanidade. Entretanto, há de se aprofundar essa temática, no sentido de verificar o saldo atual dessas transformações, sob a ótica do desenvolvimento sustentável, a fim de se adotar medidas corretivas, caso os impactos estejam propiciando maiores riscos do que oportunidades, ou um déficit em desfavor da humanidade e da preservação ambiental frente ao progresso que se impõe nas relações sociais, políticas e econômicas, sendo essa a mais importante das discussões aqui apontadas. 


\section{Conclusão}

As teorias da administração guardam interface com os aspectos econômicos, políticos e sociais vigentes em suas respectivas épocas, como se pode observar sobre a linha teórica direcionada às questões fundamentais anteriormente abordadas, onde é possível destacar alguns estudos que corroboram com esta percepção, quais sejam: i) as tecnologias digitais podem maximizar a eficiência e a produtividade dos recursos para a EC. (Eivind et al. 2020); ii) é necessário a adoção de um padrão de diretrizes organizacionais, a fim de que se possa migrar da economia linear para a EC. (British Standard (2017); iii) a EC é definida como aquela “economia regida por princípios restaurativos e regenerativos, que visa manter produtos, componentes e materiais em seus máximos valores de utilidade, distinguindo os ciclos técnicos e biológicos." (British Standard BS 8001-2017).

Quanto aos aspectos referentes à Economia Circular - EC, os estudos da Tabela 4 apontam para a prática dos 6 Rs reduzir, reciclar, reutilizar, reparar, remanufaturar e redesenhar; onde os benefícios diretos que a era tecnológica da Indústria 4.0 pode oferecer são: economia de tempo, de recursos e de custos; diminuição de riscos inclusive os de acidente; maior segurança para empregados, acionistas, clientes e consumidores, através da transparência e governança; diminuição de desperdícios e a consequente melhor gestão dos resíduos; melhoria no ciclo de vida dos produtos e serviços; efetividade na análise de impactos ambientais, seus riscos e consequências; melhoria da gestão, planejamento e produção; eficácia na logística reversa favorecendo a melhor gestão da cadeia de suprimentos, a diversificação e a relação com mercados e fornecedores; aperfeiçoamento na gestão de recursos humanos; melhor avaliação do nível de satisfação percebida e sentida pelos clientes e aperfeiçoamento no processo de decisão; favorecimento às inovações nos modelos de negócios inteligentes e sustentáveis, dentre outros. Dessa forma, as tecnologias mais citadas pelos autores das obras descritas neste estudo são: Internet das Coisas - IoT, Internet de Serviços - IoS, computação em nuvem, Big Data, Sistemas Cibernéticos Físicos - CPS, Inteligência Artificial - AI, Sistemas Ciber-Físicos de Produção - CPPS, design virtual, blockchain, impressão 3D, robótica, nanotecnologia, tecnologias de visualização (realidade virtual e aumentada), manufatura aditiva, digital gêmeos. A comunhão desses fatores da Indústria 4.0 e da EC praticados pelas organizações leva à melhoria da qualidade de vida, à melhor decência das rotinas de trabalho, à melhoria nas relações comerciais, sociais e ambientais. Exemplo desses resultados aqui citados, estão expressos em alguns estudos abaixo nomeados. 
Tabela 5. Exemplificação de contribuições ao tema estudado.

\begin{tabular}{|c|c|c|}
\hline Nome do Estudo ou da Obra & Autores & Contribuições ao tema \\
\hline $\begin{array}{l}\text { Industry } 4.0 \text { and sustainable development: } \\
\text { A systematic mapping of triple bottom line, } \\
\text { Circular Economy and Sustainable } \\
\text { Business Models perspectives. }\end{array}$ & $\begin{array}{l}\text { Iqra Sadaf Khan; Muhamada Ovais } \\
\text { Ahmad; Jukka Majava. 2021. }\end{array}$ & $\begin{array}{l}\text { A Indústria } 4.0 \text { permeada com a EC } \\
\text { aperfeiçoa a inovação e a lucratividade e } \\
\text { cria escala de valor na TBL. }\end{array}$ \\
\hline $\begin{array}{l}\text { How the combination of Circular Economy } \\
\text { and Industry } 4.0 \text { can contribute towards } \\
\text { achieving the Sustainable Development } \\
\text { Goals. }\end{array}$ & $\begin{array}{l}\text { T E T Dantas; E. D. de Souza; S. R. Soares. } \\
2020 .\end{array}$ & $\begin{array}{l}\text { O nexo entre EC e Indústria } 4.0 \text { é } \\
\text { fundamental na integração das novas } \\
\text { tecnologias e nos modelos de negócios } \\
\text { baseados em EC. }\end{array}$ \\
\hline $\begin{array}{l}\text { Potentials of industry } 4.0 \text { for supply chain } \\
\text { management within the triple bottom line } \\
\text { of sustainability - A systematic literature } \\
\text { review. }\end{array}$ & Hendrik Birkel e Julian M. Müller. 2020. & $\begin{array}{l}\text { A visão holística é necessária para suprir } \\
\text { lacunas na prática tecnológica, } \\
\text { principalmente dos pequenos negócios. }\end{array}$ \\
\hline $\begin{array}{l}\text { Industry } 4.0 \text { and sustainability: Towards } \\
\text { conceptualization and theory. }\end{array}$ & $\begin{array}{l}\text { Mirjam Beltrami; Guido Orzes e Marco } \\
\text { Sartor. 2021. }\end{array}$ & $\begin{array}{l}\text { A prontidão organizacional e tecnológica } \\
\text { como moderador de impactos. }\end{array}$ \\
\hline $\begin{array}{l}\text { Industry } 4.0 \text { ten years on: A bibliometric } \\
\text { and systematic review of concepts, } \\
\text { sustainability value drivers, and success } \\
\text { determinants. }\end{array}$ & $\begin{array}{l}\text { Morteza Ghobakhloo; Massod Fathi e } \\
\text { Manuel E. Morales. 2021. }\end{array}$ & $\begin{array}{l}\text { As tecnologias personalizam produtos, } \\
\text { vivenciam as experiências do consumidor } \\
\text { através da era "gêmeo digital". }\end{array}$ \\
\hline $\begin{array}{l}\text { A review of emerging industry } 4.0 \\
\text { technologies in remanufacturing. }\end{array}$ & Mairi Kerin e Duc Truong Pham. 2019. & $\begin{array}{l}\text { IoT e rede integrada gerando ciclo virtuoso } \\
\text { de produtos, processos, tecnologias e } \\
\text { organizações, sendo a escassez de insumos } \\
\text { um incentivo para a implementação da EC. }\end{array}$ \\
\hline $\begin{array}{l}\text { Digitalisation and intelligent robotics in } \\
\text { value chain of circular economy oriented } \\
\text { waste management-A review. }\end{array}$ & R. Sarc; A. Curtis e R. Pmberger. 2019. & $\begin{array}{l}\text { O objetivo geral da EC é o uso mais } \\
\text { eficiente de recursos e as tecnologias da } \\
\text { Indústria } 4.0 \text { são constructos para a melhor } \\
\text { gestão de resíduos, via sensores, robótica e } \\
\text { integração de máquinas e de sistemas. }\end{array}$ \\
\hline $\begin{array}{l}\text { A literature review on circular economy } \\
\text { adoption in the manufacturing sector. }\end{array}$ & Federica Acerbi e Marco Taisch. 2020. & $\begin{array}{l}\text { A padronização de dados como fator } \\
\text { facilitador para a adoção da manufatura } \\
\text { circular. }\end{array}$ \\
\hline
\end{tabular}

Fonte: Autores (2021), a partir da pesquisa no Science Direct.

De acordo com a obra Indústria 4.0 e Sustentabilidade: em direção à conceituação e teoria, de Beltrami et al., (2021), a maioria dos estudos por eles analisados são baseados nas evidências práticas e na interpretação, assim eles fazem diversas proposições entre as quais destaca-se: i - o impacto direto e indireto da indústria 4.0 nas práticas de sustentabilidade, tanto positivos quanto negativos nos ambientes sociais, naturais e econômicos; ii - esses impactos podem ser moderados pela prontidão organizacional e tecnológica; iii - a sustentabilidade deve ser elemento motivador para a indústria 4.0 se aperfeiçoar.

De acordo com Khan et al., (2021), muitos autores têm procurado desenvolver estudos sobre os fatores interrelacionados da indústria 4.0 e da EC, com ênfase no setor de manufatura, cadeia de suprimentos e na IoT. Eles indicam que permear as tecnologias da indústria 4.0 com os princípios da EC serviria para aperfeiçoar a inovação e a lucratividade em diversos setores ainda pouco explorados, como a agricultura, criando uma escala de valor com base na TBL e garantindo benefícios sociais, econômicos e ambientais.

Complementarmente, Ted Dantas et al., (2020), demonstram que o nexo entre EC e indústria 4.0 beneficia diretamente a seis dos dezessete objetivos do desenvolvimento sustentável - ODS, sendo eles: ODS 7, ODS 8, ODS, 9, ODS, 11, ODS 12 E ODS 13. Destacam ainda, que este nexo é fundamental pela integração entre tecnologias inovadoras e por modelos de negócios redefinidos com base nas atuais exigências da humanidade em relação à EC.

Birkel Hendrick et al., (2021) trazem uma visão holística na interpretação sobre as contribuições da indústria 4.0 para a EC, verificando lacunas nos estudos sobre a implementação prática dos princípios e instrumentos necessários à construção de novos modelos de negócios e no gerenciamento da cadeia de suprimentos. É preciso dotar, principalmente as organizações de pequeno porte de instrumentos capazes de viabilizar a gestão da boa prática sustentável. 
Ghobakhloo Morteza et al., (2021), no artigo - Indústria 4.0 dez anos depois: Uma revisão bibliométrica e sistemática de conceitos, impulsionadores de valor de sustentabilidade e determinantes de sucesso; caracterizam a importância da entrega de produtos e serviços personalizados, feitos à medida da necessidade de cada consumidor, vez que as tecnologias da indústria 4.0 permitem o profundo conhecimento sobre as experiências de consumo, trazendo uma realidade única. Eles denominam a era da instância virtual - gêmeo digital, na qual os sensores tecnológicos capturam informações sobre hábitos e transmitem informações integradas para a criação, produção e aperfeiçoamentos de produtos e serviços adequados a realidade individual das pessoas. $\mathrm{Na}$ avaliação descritiva dos 745 artigos por eles estudados sobre a temática da indústria 4.0, fica registrado que mais de 50\% foram produzidos em 2020, o que denota o crescente interesse da sociedade em relação a este assunto. Porém, registram uma mudança de paradigma em sua conceituação, saltando do chão de fábrica para conceitos focados em cadeia de valor, traduzida em princípios vitais de design, tempestividade, interoperabilidade, descentralização e comunhão com os fatores da EC, embora mais de $60 \%$ dos benefícios exalados nos estudos pesquisados sobre a evolução da indústria 4.0 ainda são de natureza econômica. Nas questões que influenciam a transformação digital, destaca-se os impactos do fluxo de capital público ou privado investido na evolução tecnológica e as determinantes políticas e legais sobre as condicionantes ambientais. A indústria 4.0 não se limita à mera digitalização corporativa e vai em direção à valorização das cadeias produtivas numa visão sistêmica, porém ainda não prioriza a preservação ambiental, apesar de contribuir para uma ação ecologicamente correta quando minimiza os resíduos, otimiza tempo, recursos e eficiência energética. Para ocorrer essa mudança de paradigma e rumar em direção ao enfrentamento do desemprego estrutural, das desigualdades regionais, do consumo excessivo e dos riscos de acidentes e desastres ambientais, se faz necessária a definição de metas em direção ao desenvolvimento sustentável, a promoção de projetos e ações compartilhadas entre organizações ultrapassando as barreiras geográficas e o compartilhamento de tecnologias entre os setores público e privado. Dentre as proposições de pesquisas futuras, indicam os autores para a necessidade de respostas sobre - como a componente ética e a gestão centrada no ser humano podem contribuir para a indústria 4.0 e para as tecnologias digitais?

Destaca-se ainda o que é estudado por kerin e Pham, (2019), que consideram como fatores de criação de valor: o produto, o equipamento, o processo e a organização. Para esses autores a tecnologia da informação é fundamental na comunicação interna para subsidiar o corpo gerencial sobre as rotinas de produção, manutenção e de procedimentos operacionais, melhorando assim a plataforma de execução e de resultados. Para tanto, há a necessidade de as tecnologias tipo IoT, inteligência artificial e a interação em rede de equipamentos serem efetivas em direção à melhoria contínua desses procedimentos. Destaca-se a importância das conexões mais próximas entre empresas e fornecedores para suprir as intercorrências de demandas e problemas da cadeia de suprimentos, melhorando os aspectos organizacionais através do desempenho e segurança de processos, advindos da aplicação das tecnologias da indústria 4.0. Salientam que os aumentos sucessivos nos custos de insumos e de matéria-prima, servirão de incentivo natural para a adoção de princípios da EC no ambiente empresarial, associados às inovações tecnológicas da indústria 4.0. Entretanto, faz-se necessário o aumento de investimentos em automação de processos, equipamentos, infraestrutura e na qualificação de pessoal, como pré-requisitos para a evolução da competência da indústria 4.0 em relação a era digital.

Registra-se que este estudo não pretendeu discorrer sobre todos os trabalhos que fizeram parte dos achados para o resultado aqui apresentado, porém teve o compromisso de apresentar a coletânea desses estudos, que de forma complementar possibilitam uma visão estratégica, organizacional e holística das contribuições da indústria 4.0 para a EC, apontando-se as tecnologias da indústria 4.0 que melhor contribuem para a implementação da EC.

Tem-se por aspectos limitantes observados neste estudo, o fato de considerar apenas uma plataforma para o ambiente da pesquisa, haja vista que a expansão para outros ambientes cientificamente reconhecidos como fontes de importantes 
trabalhos divulgados sobre o tema, poderiam dar maior amplitude à amostra e aos resultados. Também se observa a lacuna sobre uma análise aprofundada em relação ao cruzamento de dados de CE e de Indústria 4.0, fato que talvez permitisse a melhor identificação da interação e nível de dependência ou de importância entre as variáveis, fatores e características de ambas as abordagens. Certamente outras percepções poderiam ser agregadas a este estudo, dada a sua relevância e magnitude para a sociedade.

Porém, trata-se aqui de reunir estudos com aspectos relevantes sobre o apoio das atuais tecnologias, enfocando definições sobre a Indústria 4.0 que ultrapassaram a simples abordagem de uma inovação meramente aditiva, para um processo inovador disruptivo que agregue valor de forma holística, com ênfase no desenvolvimento econômico, social e ambiental, através do fortalecimento estrutural da CE nas organizações.

\section{Referências}

Ardito, L., Petruzzelli, A. M., Panniello, U., \& Garavelli, A. C., (2019). Towards Industry 4.0: mapping digital technologies for supply chain managementmarketing integration. Bus. Process Manag. J. 25 (2), 323e346.

Bardin, L. (2006). Análise de conteúdo (L. de A. Rego \& A. Pinheiro, Trads.). Edições 70. (Obra original publicada em 1977).

Bardin, L. L'ére logique. Robert Laffont, (1977).

Bauer, J. M., (2018). The Internet and income inequality: socio-economic challenges in a hyperconnected society. Telecommun. Policy 42 (4), 333 e343.

Beier Grischa, U. A., Niehoff Silke, R. Malte, \& Habich M. (2020). Industry 4.0: How it is defined from a sociotechnical perspective and how much sustainability it includes - A literature review. https://doi.org/10.1016/j.jclepro.2020.120856.

Beltrami, M.; Orzes G., \& Marco S. (2021). Industry 4.0 and sustainability: Towards conceptualization and theory. https://doi.org/10.1016/j.jclepro.2021.127733.

Birkel, H., \& Müller, J. M. (2020). Potentials of industry 4.0 for supply chain management within the triple bottom line of sustainability - A systematic literature review. https://doi.org/10.1016/j.jclepro.2020.125612.

British S. B. S 8001:(2017). Framework for implementing the principles of the circular economy in organizations Guide. The British Standard Institution, 2017.

Buer, S.-V., Strandhagen, J. O., \& Chan, F. T. (2018). The link between Industry 4.0 and lean manufacturing: mapping current research and establishing a research agenda. Int. J. Prod. Res. 56 (8), $2924 \mathrm{e} 2940$.

Castelo-Branco, F. \& Cruz-Jesus, T. O. (2019). Avaliando a prontidão da indústria 4.0 na manufatura: evidências para a União Europeia. Comput. Ind., 107, $22 \mathrm{de}-32,10.1016 / \mathrm{j}$. compind.2019.01.007.

Cayzer, S., Griffiths, P., \& Beghetto, V. (2017). Design of indicators for measuring product performance in the circular economy. Int. J. Sustain. Eng. 10, 289e298. https:// doi.org/10.1080/19397038.2017.1333543.

Chizzotti, A. (2006). Pesquisa em ciências humanas e sociais (8a ed.). Cortez.

Creswell, J. W. (2007). Projeto de pesquisa: métodos qualitativo, quantitativo e misto (2a ed., L. de O. Rocha, Trad.). Artmed. (Obra original publicada em 2003).

Eivind, K., Fenna, B., Patrick, M., e Jingyue, L. (2020). The smart circular economy: A digital-enabled circular strategies framework for manufacturing companies. Norwegian University of Science and Technology, NO-7491 Trondheim, Norway. University of Hamburg, 20146 Hamburg, Germany.

Ella, J., Ruud, B., \& Ingemarsdotter, E. (2020). Opportunities and challenges in IoT-enabled circular business model implementation - A case study Emilia Ingemarsdotter. Faculty of Industrial Design Engineering, Delft University of Technology, 2628 CE Delft, the Netherlands. 10.1016/j.resconrec.2020.105047.

Flick, U. (2009). Introdução à pesquisa qualitativa (3a ed.), Artmed. (Obra original publicada em 1995).

Freitas, H. M. R., Cunha, M. V. M., Jr., \& Moscarola, J. (1997). Aplicação de sistemas de software para auxílio na análise de conteúdo. Revista de Administração da USP, 32(3), 97-109.

Geng, Yong, Fu, J., Sarkis, J., \& Xue, B., (2012). Towards a national circular economy indicator system in China: an evaluation and critical analysis. J. Clean. Prod. 23, 216e224. https://doi.org/10.1016/j.jclepro.2011.07.005.

Ghobakhloo, M., Massod, F., \& Manuel, E. M. (2021). Industry 4.0 ten years on: A bibliometric and systematic review of concepts, sustainability value drivers, and success determinants. https://doi.org/10.1016/j.jclepro.2021.127052.

Ghobakhloo, M. (2020). Industry 4.0, digitization, and opportunities for sustainability. Journal of Cleaner Production, 252 119869. https://doi.org/10.1016/j.jclepro.2019.117805. 
Research, Society and Development, v. 10, n. 16, e186101622912, 2021

(CC BY 4.0) | ISSN 2525-3409 | DOI: http://dx.doi.org/10.33448/rsd-v10i16.22912

Iqra, S. K.; Muhamada, O. A.; \& Jukka, M. (2021). Industry 4.0 and sustainable development: A systematic mapping of triple bottom line, Circular Economy and Sustainable Business Models perspectives.

J-Rincon-Moreno., M. Ormaz, M. J., \& Alvarez, C. J. (2020). Universidad de Navarra, TECNUN, School of Engineering, San Sebastian, Spain. Advancing circular economy performance indicators and their application in Spanish companies. 10.1016/j.jclepro.2020.123605.

Mairi, K., \& Duc, T. P. (2019). A review of emerging industry 4.0 technologies in remanufacturing, Journal of Cleaner Production, 237, 2019, 117805

Mendoza, J. M. F., Sharmina, M., Gallego-Schmid, A., Heyes, G., \& Azapagic, A. (2017). Integrating backcasting and eco-Design for the circular economy. The BECE Framework. J Ind Ecolm 21(3):526-544.

Mozzato, A. R., \& Grzybovski, D. (2011). Análise de conteúdo como técnica de análise de dados qualitativos no campo da administração: potencial e desafios. RAC, 15(4), 731-747.

Niero, M., \& Haushild, M. Z. (2017). Closing the loop for packaging: finding a framework to operationalize circular economy strategies. Procedia CIRP 2017; 61: $685-690$.

Ormazabal, M., Prieto-Sandoval, V., Puga-Leal, R. \& Jaca, C., (2018). Circular Economy in Spanish SMEs: challenges and opportunities. J. Clean. Prod. Pp 185, 157 e 167. https://doi.org/10.1016/j.jclepro.2018.03.031.

Pigosso D. C. A., Rozenfeld H. \& Mcaloone, T. (2013). Ecodesign Maturity Model: A Management Framework to Support Ecodesign Implementation into Manufacturing Companies. J. Clean Prod. 2013; 59: 160-173.

Schroeder, A., Ziaee Bigdeli, A., Galera Zarcos, C. \& Baines, T., (2019). Capturing the benefits of industry 4.0: a business network perspective. Prod. Plan. Control e 117.

Aquino, A. R. et al. Sustentabilidade ambiental. OUERJ, 2015. 167 p.: il.

Ted Dantas, E. D., de Souza, I. R., Destro, G., Hammes, C. M. T., \&Rodrguez, S. R. S. (2020). How the combination of circular economy and industry 4.0 can contribute towards achieving the sustainable development goals. https://doi.org/10.1016/j.sps.2020.10.005.

Weetmen, C. (2019). The circular economy handbook for business and supply chains: repair, remake, redesign, rethink; tradução Afonso Celso da Cunha Serra. Autêntica Business.

World Economic Forum. 2014. <WF_Annual_Report_2014_15.pdf (weforum.org)>. 